Paper ID \#17961

\title{
The proposed approach for determining combined stresses of a component
}

\section{Dr. Xiaobin Le P.E., Wentworth Institute of Technology}

Professor, Ph.D, PE., Department of Mechanical Engineering and Technology, Wentworth Institute of Technology, Boston, MA 02115, Phone: 617-989-4223, Email: Lex@ wit.edu, Specialization in Computer Aided Design, Mechanical Design, Finite Element Analysis, Fatigue Design and Solid Mechanics

\section{Prof. Anthony William Duva P.E. P.E., Wentworth Institute of Technology}

Anthony W. Duva An Associate Professor in the Mechanical Engineering and Technology Department at Wentworth Institute of Technology since 2001 with 14 years of prior full time industrial experience. He has worked in the design of various technologies from advanced underwater and ultrahigh altitude propulsion systems to automated manufacturing equipment. His interests include advanced thermal and mechanical system design for green power generation.

\section{Prof. John Voccio, Wentworth Institute of Technology}

Assistant Professor, Ph.D, Department of Mechanical Engineering and Technology, Wentworth Institute of Technology, Boston, MA 02115, Phone: 617-989-4258, Email: voccioj@wit.edu, Specialization in Mechanical Design, Finite Element Analysis and Superconducting Magnets

\section{Prof. Richard L Roberts, Wentworth Institute of Technology}

Associate Professor, Department of Mechanical Engineering and Technology, College of Engineering and Technology, Wentworth Institute of Technology, 550 Huntington Ave., Boston, MA 02115

\section{Prof. Ali Reza Moazed, Wentworth Institute of Technology}

Ali R. Moazed is a Professor in the Mechanical Engineering and Technology department at Wentworth Institute of Technology. In addition to over thirty years of industrial and consulting experience, he has taught mechanical engineering courses full or part-time prior to joining Wentworth in 2001. At Wentworth, he teaches design related courses in the solid mechanics area. He believes in teaching from the perspective of a practicing academician by bringing into the classroom topics related to the practice of engineering, along with the latest pedagogical tools.

His expertise is in the area of Applied Finite Element Analysis (FEA) and as an engineering consultant, he provides FEA services to the Utility, Industrial, and Commercial clients nationwide. These services include design analysis, design verification, design modification, design optimization, and failure analysis. $\mathrm{He}$ is a registered professional engineer in states of Massachusetts and California. 


\title{
The Proposed Approach for Determining Combined Stresses of a Component
}

\begin{abstract}
For a mechanical engineering program, one of the main learning outcomes is that students can design mechanical system components. Achieving this outcome requires students be trained to calculate the combined stresses of a component with multiple loadings by using typical theoretical formulas. We have found, however, that students had difficulty in effectively calculating these combined stresses. We believe that this was mainly due to some misconceptions regarding combined stress. During the last several years, we proposed and gradually modified an approach which facilitated students to calculate stress under combined loading. The proposed approach contains 7 steps which clearly demonstrate the relationships between the forces and the induced stresses. The process guides students in the calculation of stress components due to each individual internal resultant force and how to correctly combine these stresses to form the state of stress at the point under the consideration. This paper will describe the proposed procedure with results of the implementation of the procedure in our teaching. The effectiveness of the proposed approach was evaluated using different classes performance on the combined stress problem in the midterm exam. In two class sections, we explained and implemented the proposed approach with three examples. In another two class sections, we just used the traditional approach; that is, we followed the topics arranged in the textbook for calculating the combined stress. The comparison of the grades on the combined stress problem indicated that the exam average score in the sections with the implementation of the proposed procedure was statistically significantly higher than the exam average score in the sections without the implementation of the procedure. A survey was conducted in the classes regarding the proposed procedure. The feedback information from the survey was very positive. This paper will also present these results of the data analysis of the student survey.
\end{abstract}

\section{Introduction}

For a mechanical engineering program, one of the main learning outcomes is that students can design machine components. To design a safe component, students are required to apply the appropriate failure theories to evaluate the factor of safety at possible weak points. In order to apply failure theories for evaluating the factor of safety, students must know the state of stress at the points of interest. These stresses are typically combined stresses in the real components resulting from several different loading conditions. In engineering practice, FEA (Finite Element Analysis) simulation is used for determining the state of stress or directly showing the safety factor distribution of the component. In academics, however, mechanical engineering students are required to be trained to perform hand calculations for determining the state of stress of the interesting points by using typical theoretical formulas.

In our mechanical engineering program, two technical courses are devoted to teaching methods required for the calculation of the state of stress; these are MECH2500-Mechanics of Materials and MECH3000-Design of Machine Elements. MECH2500-Mechanics of Materials focuses on how to calculate the stress induced by simple individual force, such as axial loading, bending 
moment, torsion and lateral shear force. At the end of the course, there is a short chapter about the state of stress caused by the combined loading [1]. At the beginning of MECH3000-Design of Machine Elements, the general state of stress caused by the combined loadings must be reviewed in detail, because a prerequisite for using the failure theory is to determine the state of stress of the point ${ }^{[2]}$. Thru our assessment process, we found that there was a common issue that students had some difficulty in effectively calculating the general state of stress of components with multiple loadings, that is, the combined stresses.

The theories and formulas for the combined stress calculation are clearly described in any textbook $^{[1,2]}$. During previous semesters, we devoted time to discussing combined stress calculations with students to determine why they had difficulty determining the combined stresses. While they knew that the calculation of combined stress was an important skill, they felt that they were "lost" when they dealt with the combined stress issue. This might be simply caused by the misconceptions about the stress because undergraduate students often have far less conceptual abstract understanding in core engineering courses than faculty assumes ${ }^{[3]}$. Many educators have used the concept inventories to investigate the possibility of misconception in the technical core course Mechanics of Materials ${ }^{[3,4]}$. They found that students had some misconceptions on normal and shear stress and deformations in axially loaded members ${ }^{[4]}$. Some educators used physical experiments and FEA (Finite Element Analysis) to help students to have a better understanding of the combined stresses ${ }^{[5,6]}$. Typically, in the course of mechanics of materials, the concepts, formulas, and procedures for combined loading are discussed in lectures towards the end of the course. Some educators suggested that the concepts, formulas, and procedure of the combined stress calculation should be presented and discussed much earlier ${ }^{[7]}$, so students have more time to comprehend the combined stress calculations.

The authors have taught the of MECH2500-Mechanics of Materials and MECH3000-Design of Machine Elements courses for many years. After we noticed that students had difficulty in the calculation of combined stresses, we have tried various methods to help students in effectively determining the state of stress over the last several years. We believed that clear descriptions of combined stress (concepts) and an executable procedure for combined stress calculation could help students have a better understanding of what the calculations indicate. During the last several years, we proposed and gradually modified an approach to facilitate students calculating the combined stress under combined loadings. The modified approach contains 7 steps, including 2 tables and 3 graphical instructions, which clearly demonstrate the relationships between the forces and the induced stresses. The method provides a guide for students in developing the stress calculation due to each individual internal resultant force and helps them to correctly combine these stresses to form the state of stress at the point of interest.

This paper will present the proposed procedure and its implementation in our teaching methodology. In order to evaluate the effectiveness of the proposed approach, we collected the scores of the combined stress problem in the midterm exam from two different classes. In two class sections, we explained and implemented the proposed approach with three examples. In another two class sections, we used the traditional approach; that is, we followed the topics arranged in the textbooks ${ }^{[1,2]}$ to determine the combined stress. After the procedure was 
implemented, a class survey was conducted to obtain feedback from students on the effectiveness of the proposed procedure.

\section{Proposed procedure for determining the combined stresses}

Typical stress formulas for a component under simple loading, including axial normal loading, torque, bending moment and shearing force (lateral force), are fully discussed and clearly demonstrated in MECH2500-Mechanics of Materials. Based on the conversations with students, we found that some students had difficulties in calculating the combined stresses for the following reasons:

(1) They had misconceptions about the combined stresses,

(2) They were not sure what stress will be induced by an applied force,

(3) They were not sure how to start the solution and necessary steps,

(4) They were not sure how to combine the induced stresses into the combined stresses.

Over the last several years, based on these findings, the authors proposed and continuously modified a procedure for calculating the combined stresses.

\subsection{The procedure}

The procedure for determining the combined stress is composed of the following 7 steps.

Step 1: Understand the general state of stress

The general state of stress of a point under the consideration is shown in the differential element of Figure 1. In order to understand the general state of stress of a point, students need to understand and recognize following concepts and facts:

- For a complete stress status of an element of the point, there are 6 independent stresses: 3 normal stresses, $\sigma_{x}, \sigma_{y}, \sigma_{z}$, and 3 shear stresses, $\tau_{x y}, \tau_{y z}, \tau_{z x}$.

- Stress is the intensity of force per unit area and is induced by an internal force generated by applied loads. If there is no internal force at the location of interest, no stress will be induced.

- On each side of the stress element, there are 3 stresses: one normal stress and two shear stresses, which are induced by the internal resultant forces on the imaginary cut plane through this side surface. The induced stresses will be determined by using the internal resultant forces on this imaginary cut plane.

- In order to get the full state of stress of the point, three imaginary cuts through the point must be made; these are parallel to the $\mathrm{x}-\mathrm{y}$ plane, $\mathrm{y}-\mathrm{z}$ plane, and $\mathrm{z}-\mathrm{x}$ plane. 


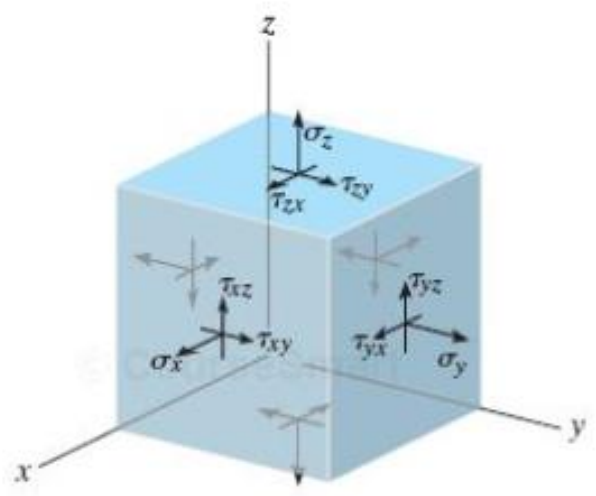

Figure 1 The general state of stress of a point under the consideration

Step 2: Determine the 6 internal forces on the imaginary cut plane through the point.

On the imaginary cut plane, for 3D problems, there are 6 internal resultant forces which will be applied to the Cartesian coordinate system at the centroid point of the cut-off cross section. Figure 2 shows 6 internal resultant forces. For 2D problems, there will be only three internal resultant forces.

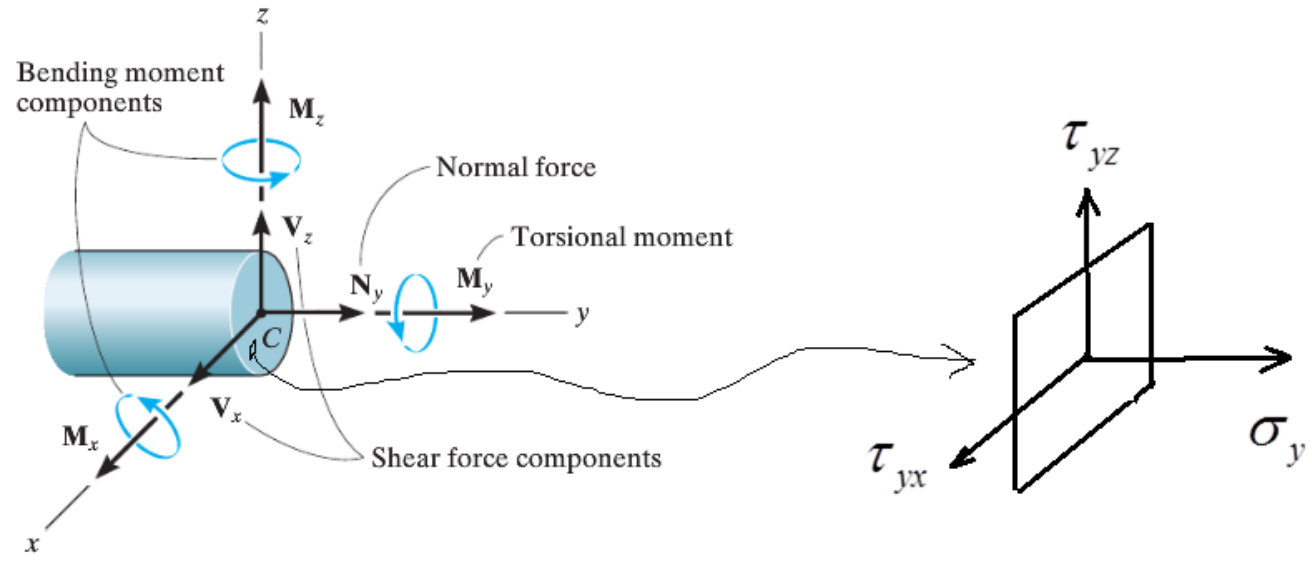

Figure 2 General 6 internal forces on a cross-section

Step 3: Understand the relations of the internal resultant force vs. the corresponding induced stress on one side of the stress element as shown in Figure 2

For the z-x cut-off plane as shown in Figure 2, one side of the stress element on the cut-off plane has 3 stresses: $\sigma_{y}, \tau_{y x}, \tau_{y z}$

- Axial loading $N_{y}$ will induce normal stress $\sigma_{y}$,

- Two bending moments, $M_{x}, M_{z}$, will also induce normal stress $\sigma_{y}$,

- The shear force $V_{x}$ will induce the shear stress $\tau_{y x}$,

- The shear force $V_{z}$ will induce the shear stress $\tau_{y z}$, 
- The torque $M_{y}$ will induce the shear stress normal to the radial line of the point following the torsion direction, which can be resolved into $\tau_{y x}$ and $\tau_{y z}$. The values of these $\tau_{y x}$ and $\tau_{y z}$ are dependent on the location of the point.

Table 1 relates the internal resultant force to the induced stress. The first row shows the 6 possible internal resultant forces on the $\mathrm{z}$-x cut-off plane along the local coordinate system through the centroid of the cut-off cross-section. The first column shows the three possible induced stresses. The "Yes" in Table 1 indicates the stress identified in column 1 will be induced by the corresponding internal force identified in the column heading. For example, the "Yes" in the cell corresponding to the $2^{\text {nd }}$ row and $2^{\text {nd }}$ column means that the normal stress $\sigma_{y}$ will be induced by the internal resultant normal force $N_{y}$. The "No" in Table 1 indicates the stress identified in column 1 will not be induced by the internal force identified in the column heading. For example, the "No" in the cell corresponding to the $3^{\text {rd }}$ row and $2^{\text {nd }}$ column indicates that the shear stress $\tau_{y x}$ cannot be induced by the internal resultant normal force $N_{y}$. Table 1 can be used as a guide for students to choose the proper stress formula for calculating the stress induced by the corresponding internal resultant forces.

Table 1 Internal resultant forces vs. corresponding induced stress

\begin{tabular}{|l|l|l|l|l|l|l|}
\hline & $N_{y}$ & $V_{x}$ & $V_{z}$ & $M_{y}$ & $M_{x}$ & $M_{z}$ \\
\hline Normal stress $\sigma_{y}$ & Yes & No & No & No & Yes & Yes \\
\hline Shear stress $\tau_{y x}$ & No & Yes & No & Yes & No & No \\
\hline Shear stress $\tau_{y z}$ & No & No & Yes & Yes & No & No \\
\hline
\end{tabular}

Step 4: Calculate the induced stresses of the point on the imaginary cut-off planes

Using Table 1 as a reference, each induced stress at the point on the imaginary cut-off plane by each internal resultant force can be calculated by using the stress formulas fully discussed and demonstrated in MECH2500-Mechanics of Materials. Taking Figure 2 as the example, the following 6 formulas can be used to calculate each induced stress.

Normal stress due to axial loading $N_{y}$ :

$$
\sigma_{y}=\frac{N_{y}}{A}
$$

Normal stress due to bending moment $M_{x}$ :

$$
\sigma_{y}=\frac{-M_{x} z}{I_{x}}
$$

Normal stress due to bending moment $M_{z}$ :

$$
\sigma_{y}=\frac{M_{z} x}{I_{z}}
$$


Shear stress due to the lateral shear force $V_{x}$

$$
\tau_{y x}=\frac{Q_{x} V_{x}}{t I_{x}}
$$

Shear stress due to the lateral shear force $V_{z}$

$$
\tau_{y z}=\frac{Q_{z} V_{z}}{t I_{z}}
$$

Shear stress due to the torque $M_{y}$

$$
\tau=\frac{M_{y} \rho}{J_{y}}
$$

The shear stress of the point calculated by the equation (6) can be dissolved into $\tau_{y x}$ and $\tau_{y z}$ at the point.

Step 5: Apply the superposition principle

If the deformation is small, that is, in the elastic region, the stresses of the component under several combined loadings are equal to the combination of the stress caused by each individual loading. But only the same type of stresses will be combined together as shown in Table 2 and Figure 3.

The combined stresses at the considered point can be calculated by using the superposition principle. Table 2 and Equations 1 thru 6 can be used as a guideline to determine the combined stresses.

Table 2 the combined stresses

\begin{tabular}{|l|l|l|l|}
\hline Induced Stress & Normal stress $\sigma_{y}$ & Shear stress $\tau_{y x}$ & Shear stress $\tau_{y z}$ \\
\hline Combined stress & $\sigma_{y}$ induced by $N_{y}$ & $\tau_{y x}$ induced by $V_{x}$ & $\tau_{y z}$ induced by $V_{z}$ \\
& $+\sigma_{y}$ induced by $M_{x}$ & $+\tau_{y x}$ induced by $M_{y}$ & $+\tau_{y z}$ induced by $M_{y}$ \\
& $+\sigma_{y}$ induced by $M_{z}$ & & \\
\hline
\end{tabular}

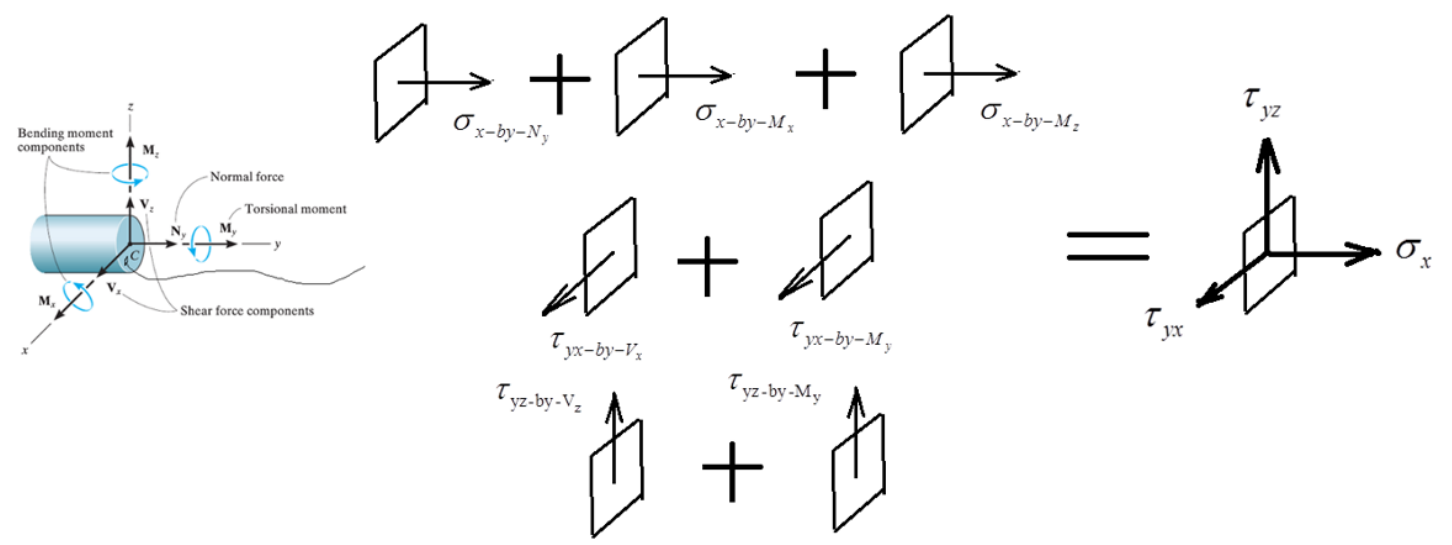

Figure 3 Visualizing the combined stresses in the $y-z$ plane 
Step 6: Repeat steps 2, 3, 4 and 5 for the $x-y$ and $y-z$ cut-off planes

For the general 3D case, the steps 2, 3, 4 and 5 are required to be repeated another two times, for a total of three cut planes through the point to assemble the 6 independent stress components. Students must be reminded, there is no induced stress if there is no internal force. If we know that there is no applied or internal force on a particular cut-off plane, the corresponding stresses are simply zero, requiring no further computation.

Step 7: Demo examples

To properly explain and demonstrate the proposed procedure, some examples should be discussed and completed during the lectures. The following three examples can be used as demonstrations.

- Example 1: 3 orthogonal cuts at the point a for a rectangular bar under axial loading as shown in Figure 4.

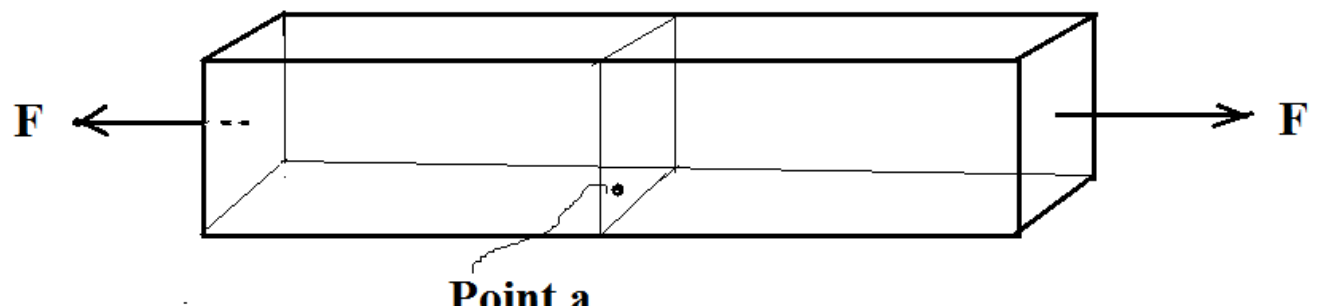

Figure 4 The state of stress at the point "a" for a bar under axial loading

- Example 2: A rectangular bar with an off-set normal force on the one end, shown in Figure 5.

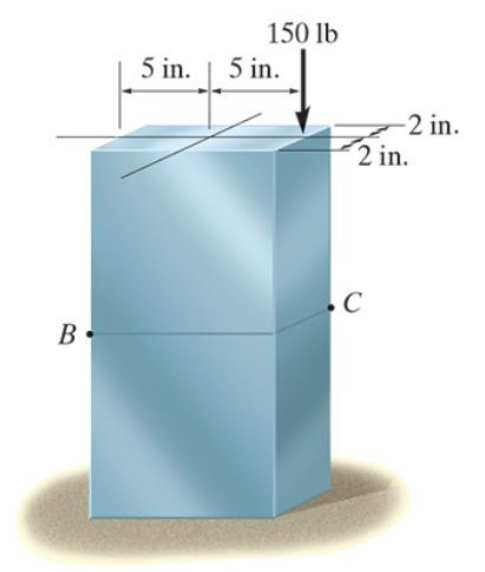

Figure 5 The state of stress at the point $\mathrm{C}^{[1]}$

- Example 3: The L-shaped round bar under a lateral load on one end, shown in Figure 6. 


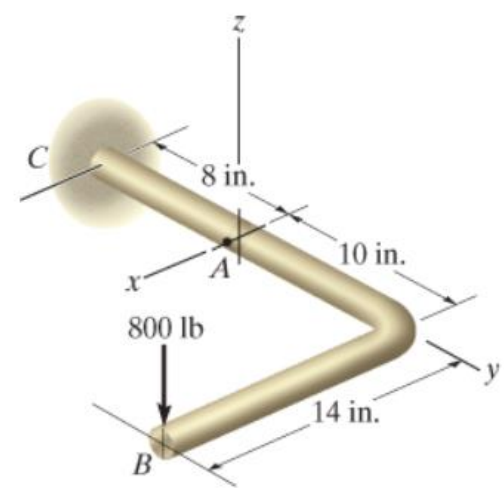

Figure 6 the state of stress at the point $A^{[1]}$

\subsection{Implementation notes for using the procedure in the classroom}

The proposed procedure for combined stress provides a concise summary of the MECH2500Mechanics of Materials because it uses all the theoretical material delivered in the course. The following are some implementation notes for using the proposed procedure:

- Step 1 "Understand the general state of stress" is used to clearly explain what is the state of stress. The clear explanation and discussion of step 1 will help students to correct their misconceptions.

- Step 2 reviews determining the internal resultant forces, which will be along the local Cartesian coordinate system through the centroid point of the cut-off cross section. Some students made mistakes in calculating the internal resultant forces on the point where the stresses are required to be calculated.

- Steps 3, 4 and 5 clearly show how to determine the type of induced stress, calculate the induced stress and combine the same types of induced stress.

- The proposed procedure was viewed as clear and manageable to our faculty group. But, in order to make sure that students can fully understand and implement it, several demonstration examples should be completed during lectures. Three examples in step 7 were demonstrated using the procedure. Example 1 is used to demonstrate that the three imaginary cuts should be made in order to get the full state of stress of the point. This example also helps students understand that the absence of an internal force will not induce stress. Example 2 will be used to show how to combine two induced normal stresses, and Example 3 will be used to show how to combine two induced shear stresses. More examples will be helpful if time permits.

\section{Implementation and data analysis}

\subsection{Implementation of the proposed procedure}

In the 2016 fall semester, we used 3 lectures to fully implement the proposed procedure for calculating combined stresses in two class sections of MECH3000-Design of Machine Elements. One lecture was used to explain the proposed procedure. Most of the lecture hour in the first lecture was used for Steps 1, 3, 5, which involved the concepts of the state of stress, the relationship of the internal resultant forces vs. the corresponding induced stress, and the 
combination of the same types of induced stresses, respectively. The two additional lectures were used to demonstrate and implement the proposed procedure to solve several examples in detail. During the implementation, the authors found that a lot of time was actually spent in reviewing material needed for step 2, calculating the internal resultant forces on the cut-off planes.

\subsection{Student performance evaluation thru direct observations}

The midterm exam for the course contained a combined stress problem. During the midterm exam, students allowed to have several sheets of notes. To assess the effectiveness of the proposed procedure, we collected the scores of the combined stress problem in the midterm exam from sections $02,03,05$ and 06 . The proposed procedure had been clearly explained, discussed and implemented in sections 05 and 06 . The proposed procedure had not been implemented in section 02 and 03, where the combined stress lectures were addressed by following the approach provided in the Hibbeler's and Shigley's textbooks ${ }^{[1,2]}$. The collected test results on the combined stress problem are shown in Table 3.

Table 3 A summary of the collected exam data on the combined stress problem

\begin{tabular}{|l|c|c|c|}
\hline Class sections & Sample size & Mean (the maximum point is 30) & Standard deviation \\
\hline $\begin{array}{l}\text { MECH3000-05 } \\
\text { MECH3000-06 }\end{array}$ & 43 & 22.65 & 5.61 \\
\hline $\begin{array}{l}\text { MECH3000-02 } \\
\text { MECH3000-03 }\end{array}$ & 48 & 18.17 & 7.44 \\
\hline
\end{tabular}

Using the $99 \%$ confidence level, the critical theoretical $t$-value is $t_{\text {theory }}=2.370$. The experimental $t$-value based on the collected exam data is $t_{\text {exam }}=3.217$. Since $t_{\text {exam }}=3.217$ is larger than $t_{\text {theory }}=2.370$, it can be concluded that the proposed procedure for calculating the combined stresses is effective because the exam average score in sections 05 and 06 are statistically significantly higher (with a confidence level 99\%) than the exam mean score in sections 02 and 03.

Even though the proposed procedure was effective, the exam mean was only 22.65 out of the maximum possible score 30 . If the percentage was used to express the exam mean, the average score was $75.5 \%$.

\subsection{Student understanding obtained thru a survey}

The class survey was administered to MECH3000-05 and MECH3000-06 for collecting student feedback for the proposed procedure and the combined stress topic. The class survey had two questions. A total of 41 students participated in the survey.

The first question was used to check whether the proposed procedure helped them to execute the combined stress calculation. The survey results are shown in Table 4. The majority of students $(68.3 \%)$ said "yes". However, $22.0 \%$ of students, which was not a small percentage, said "no." 
Table 4 the survey results on question \#1

Question \#1: "The procedure of determining the combined stress discussed in the lectures facilitates me to run the calculation of the combined stress"

\begin{tabular}{|l|c|c|l|l|}
\hline Choice & MECH3000-05 & MECH3000-06 & Total & Percentage \\
\hline Strongly agree & 0 & 1 & 1 & $68.3 \%$ \\
\hline Agree & 14 & 13 & 27 & \\
\hline No opinion & 2 & 2 & 4 & $9.7 \%$ \\
\hline Disagree & 5 & 4 & 9 & $22.0 \%$ \\
\cline { 1 - 4 } Strongly disagree & 0 & 0 & 0 & \\
\cline { 1 - 3 }
\end{tabular}

The second question was used to check whether the proposed procedure helped them develop a better understanding of the combined stress calculation. The survey result is shown in Table 5. The majority of students (68.3\%) said "yes". However, $22.0 \%$ of students, which was not a small percentage, again said "no."

Table 5 the survey results on question \#2

Question \#2: "The procedure of determining the combined stress discussed in the lectures facilitates me to have better understanding of determining the combined stress"

\begin{tabular}{|l|c|c|l|l|}
\hline Choice & MECH3000-05 & MECH3000-06 & Total & Percentage \\
\hline Strongly agree & 0 & 1 & 1 & $68.3 \%$ \\
\hline Agree & 12 & 15 & 27 & \\
\hline No opinion & 2 & 2 & 4 & $9.7 \%$ \\
\hline Disagree & 6 & 2 & 8 & $22.0 \%$ \\
\cline { 1 - 4 } Strongly disagree & 1 & 0 & 1 & \\
\cline { 1 - 3 }
\end{tabular}

\section{Discussions and conclusions}

In practice, most mechanical components typically have multiple forces applied and the state of stress at the potential weak point is always a combined stress condition. In academics, it is therefore very important that mechanical engineering students understand how to calculate combined stresses.

Over the last several years of teaching, it was found that some students had difficulty in effectively calculating these combined stresses. It could be caused by a lot of different reasons. Based on conversations with students and faculty regarding combined stress calculations, we found 4 common issues:

(1) They had misconceptions about the combined stresses,

(2) They were not sure what stress will be induced by an applied force,

(3) They were not sure how to start the solution and necessary steps

(4) They were not sure how to combine the induced stresses into the combined stresses.

The theoretical formula and procedure for calculating the combined stress are clearly described in any textbook. However, the procedures in these textbooks ${ }^{[1,2]}$ don't have some visual help or 
some tables to clearly and directly display the relationships between the internal forces and the induced stresses. The proposed procedure in this paper provides clear descriptions of the concepts and executable steps. Furthermore, the proposed procedure contains two visual sketches and two tables for clearly indicating and explaining the relationships between the internal forces and the induced stresses. Some students told us that these visual sketches and tables helped them to create the connections in their minds between the forces and stresses for the calculation of the combined stress.

The comparison of the exam scores of the combined stress problem of the midterm exam in the classes with and without implementation of the proposed procedure indicated that the proposed procedure was effective and improved the exam scores by a statistically significant margin. The class survey also indicated that the majority of students $(68.3 \%)$ had positive feedback about the proposed procedure and agreed that the proposed procedure facilitated them to conduct the calculation of the combined stress and helped them to have a better understanding of combined stress.

\section{References}

[1].R.C. Hibbeler, Mechanics of Materials; 10 Edition, Prentice Hall; 2013

[2].Richard G. Budynas and J. Keith Nisbett, “Shigley's Mechanical Engineering Design”, 10 ${ }^{\text {th }}$ Edition, McGraw Hill 2016.

[3].Brown, S., \& Montfort, D., \& Findley, K. (2007, June), Student Understanding Of States Of Stress In Mechanics Of Materials Paper presented at 2007 Annual Conference \& Exposition, Honolulu, Hawaii.

[4]. Brown, S., \& Lewis, D. (2010, June), Student Understanding Of Normal And Shear Stress And Deformations In Axially Loaded Members Paper presented at 2010 Annual Conference \& Exposition, Louisville, Kentucky.

[5].Coyle, M., \& Keel, C. (2001, June), A Combined Stress Experiment Using A Hacksaw Paper presented at 2001 Annual Conference, Albuquerque, New Mexico.

[6]. Szaroletta, W. (2002, June), Enhancing Learning Opportunities In A Combined Stress Laboratory Paper presented at 2002 Annual Conference, Montreal, Canada.

[7]. Sadid, H., \& Wabrek, R. (2009, June), A New Approach To Teaching Mechanics Of Materials Paper presented at 2009 Annual Conference \& Exposition, Austin, Texas. 